\title{
Profesionalización de la Gestión Sanitaria basada en los resultados a través de la Educación a Distancia y formación basada en la simulación
}

Desarrollo de un simulador para mejorar la gestión en los hospitales y centros de salud

Professionalization on Result-based Healthcare Management through Distance Education and Simulation-Based Training / LASALUS

Equipo UNIBE-ParaguaY:

- Mónica Ruoti.

- Raquel Araujo.

- Daniel Franco.

- Hugo Sperati.

- Eduardo Velázquez.

El proyecto LASALUS, está cofinanciado por la Comunidad Europea en el marco del Programa Erasmus +. Tendrá una duración de tres años y se inició el 24 de febrero del 2015 en la Ciudad de Buenos Aires donde se realizó el acto internacional de apertura del Proyecto

PaRTICIPAN CUATRO UNIVERSIDADES EUROPEAS Y SEIS LATINOAMERICANAS

- Università degli Studi di Pavia (Italia)

- Universitá degli Studi di Roma La Sapienza (Italia)

- Ecole des Hautes Études en Santé Publique (Francia)

- Universidad Pública de Navarra (España)

- Universidad de Buenos Aires (Argentina)

- Universidad ISALUD de Argentina

- Universidad Evangélica (EI Salvador)

- Universidad Nueva San Salvador (El Salvador)

- Universidad Iberoamericana (Paraguay)

- Universidad Gran Asunción (Paraguay)

Que trabajarán junto al Consorcio de Bioingeniería e Informática Médica (CBIM) de Italia para desarrollar un simulador inédito en gestión de salud orientado a la profesionalización de los administradores de las organizaciones que proveen servicios de cuidados de salud en América y Europa.

Apoyan también Al Proyecto LASALUS la Organización Iberoamericana de la Seguridad Social (OISS), El Ministerio de Salud de Italia, la Federación Argentina de Trabajadores de la Sanidad (FATSA), los Hospitales Garrahan y El Cruce de Argentina, y la Fundación CIRNA ONLUS de Pavía. 
Profesionalización de la Gestión Sanitaria basada en los resultados a través de la Educación a Distancia y formación basada en la simulación

\section{OBjetIVO GENERAL}

"Contribuir a la consecución de los MDG en los países de Latinoamérica (LA), el desarrollo de un crecimiento socioeconómico sostenible e integrador, así como la cohesión social y la equidad. Aumentar, a través de un mejor acceso a los servicios de mayor calidad y de atención de salud eficiente, con una mejor gestión."

\section{Objetivos ESPECÍfICOS}

- Aumentar y mejorar el acceso a la Educación superior de los profesionales de LA que trabajan en las instituciones de la salud a través del desarrollo e implementación del nuevo plan de estudios (Diplomado, Especialización, Maestría) en la gestión de la asistencia sanitaria mediante la Educación a Distancia y la formación basada en la simulación.

- Mejorar el nivel de competencias y habilidades en LA IES para el diseño e implementación de los planes de estudios de postgrado con componentes de e-learning y la formación basada en la simulación.

\section{ACTIVIDADES PRINCIPALES}

1.2 Recolección de información de las necesidades del usuario.

1.3 Análisis de las necesidades del usuario.

1.4 Desarrollo de análisis de la situación.

1.6 Elaboración y publicación de los documentos de antecedentes.

2.1 La construcción por instituciones de Educación superior Europea de un catálogo de buenas prácticas y lecciones aprendidas.

2.2 Preparación y desarrollo de una pasantía.

2.3 Talleres para la difusión del contenido aprendido por los internos.

2.4 Preparación de directrices orientadores y dispositivos de apoyo.

3.1 Desarrollo del lado del servidor y el cliente del software de simulación.

3.2 Definición de equipamiento tecnológico para el servidor en LA IES áreas de e-learning.

3.4 Desarrollo y de la versión Beta del CSHCMT.

3.6 Monitoreo y probar la versión final de CSHCMT.

3.7 Desarrollo de casos de simulación para el CSHCMT;

3.8 Monitoreo CSHCMT: utilización en cursos y carreras.

4.1 Talleres para la elaboración de los planes de estudio de línea de base.

4.2 Elaboración de los 'currículos clúster gestión sanitaria de línea de base 


\section{Proyectos de la UNIBE}

4.3 Diseño del plan de estudios personalizado.

4.4 Elaboración del plan de estudios.

4.5 Elaboración de material didáctico.

4.6 Elaboración de recursos audiovisuales.

5.1 Preparación de un curso de formación modular para el personal de LA HIEs y profesores.

5.6 Evaluación de la segunda etapa del curso de formación (profesores).

6.1 La aprobación del nuevo plan de estudios por las autoridades legales.

6.2 Difusión de la nueva oferta formativa.

6.3 Designación de los maestros.

6.6 Desarrollo del primer año del curso.

6.7 Evaluación del proceso de formación.

7.1 Elaboración de un plan de difusión.

7.2 Creación de una página web del proyecto.

7.5 Elaboración de 6 boletines.

7.6 El diseño y la impresión de material gráfico.

7.8 Cobertura de los medios sociales.

8.1 El establecimiento de estándares de calidad predeterminados: plan de calidad.

8.5 Elaboración de informes de control de calidad del proyecto.

9.1 Formalización de la red y los acuerdos entre las IES participantes.

9.7 Monitoreo, y control evaluación de cualquier desviación en el avance del proyecto.

\section{Etapas del Proyecto}

Primera Etapa: Relevamiento de la Información (Investigación para el análisis de la situación)

- 1er. Work package (WP n¹): Momento analítico ( investigación)

Segunda Etapa: Transferencia de conocimientos y el diseño y desarrollo de un simulador

- 2er. Work package (WP n²): Transferencia de conocimientos

- 3er. Work package (WP n³): Diseño y desarrollo del simulador

- 1er. Work package (WP $\left.n^{\circ} 4\right)$ : Construcción de las bases curriculares regionales 
Profesionalización de la Gestión Sanitaria basada en los resultados a través de la Educación a Distancia y formación basada en la simulación

En la primera etapa del proyecto ya ejecutada en el 2016, se ha realizado una investigación de línea de base con el objetivo de "Identificar necesidades, percepciones e intereses de actores claves de grupos involucrados que sirvan de referencia para la construcción de las bases curriculares regionales para la profesionalización de la gestión sanitaria en Latinoamérica".

Para lo cual el equipo de trabajo de la UNIBE procedió a identificar a los actores, instituciones o personas representantes del país de los distintos grupos de interés en relación a la formación en gestión sanitaria para obtener información sobre las actitudes opiniones y experiencias de los distintos grupos de actores considerando los siguientes campos, dimensiones de análisis:

\section{DIMENSIONES-CATEGORIAS DE ANALISIS}

\begin{tabular}{|c|c|c|}
\hline Campo de Análisis & Dimensiones & Actores \\
\hline \multirow{2}{*}{ INSTITUCIONAL } & E-Lerning & $\begin{array}{l}\text { Participantes del Proyecto y } \\
\text { referentes de la IES:UNIBE }\end{array}$ \\
\hline & $\begin{array}{l}\text { Formación en } \\
\text { Gestión Sanitaria }\end{array}$ & $\begin{array}{l}\text { Participantes del Proyecto y } \\
\text { referentes de la IES:UNIBE }\end{array}$ \\
\hline \multirow{2}{*}{ EDUCATIVO } & $\begin{array}{l}\text { Desarrollo } \\
\text { Curricular en } \\
\text { Gestión Sanitaria }\end{array}$ & $\begin{array}{ll}\text { - } & \text { Secretarios } \\
\text { - } & \text { Académicos } \\
\text { - } & \text { Directores } \\
\text { - } & \text { Coordinadores } \\
\text { - } & \text { Docentes } \\
\text { - } & \text { Graduados de carreras de } \\
& \text { Gestión sanitaria }\end{array}$ \\
\hline & $\begin{array}{l}\text { Procesos de } \\
\text { Enseñanza y de } \\
\text { Aprendizaje en } \\
\text { Gestión Sanitaria }\end{array}$ & $\begin{array}{ll}\text { - } & \text { Directores } \\
\text { - } & \text { Coordinadores } \\
\text { - } & \text { Docentes } \\
\text { - } & \text { Alumnoduados de carreras de } \\
& \text { Gestión sanitaria }\end{array}$ \\
\hline LABORAL & $\begin{array}{l}\text { Formación en } \\
\text { Gestión Sanitaria }\end{array}$ & $\begin{array}{ll}\text { - } & \text { Autoridades sanitarias } \\
\text { - } & \text { Directores de Hospitales } \\
\text { - } & \text { Oúblicos y Privados) } \\
& \text { acreditación de Instituciones } \\
& \text { sanitarias } \\
\text { - } & \text { Asociación de profesionales } \\
\text { - } & \text { Organizaciones de la } \\
\text { - } & \text { seguridad social } \\
& \text { Entidades gremiales }\end{array}$ \\
\hline
\end{tabular}




\section{Proyectos de la UNIBE}

El tipo de estudio desarrollado es observacional descriptivo de enfoque cualitativo. Se realizaron seis entrevistas, entrevistas semi-estructuradas, un grupo focal y revisión documental.

Se identificaron actores e instituciones del país de dos grupos de interés, el primero vinculado a la profesionalización de la gestión sanitaria (alumnos, docentes, gestores, autoridades educativas y de salud) y el segundo compuesto por el personal académico de la UNIBE.

Parte de la segunda etapa "Transferencia de Conocimientos" se ha cumplido con la pasantía de tres profesionales de la UNIBE en la Universidad de Navarra (Pamplona- España), Escuela de Altos Estudios en Salud Pública (Rennes-Francia) y la Universidad de Pavia (Italia) quienes a lo largo de tres semanas (26 de junio- al 15 de julio 2016), compartieron con los profesionales de las demás universidades participantes recibiendo capacitación, a través de talleres, seminarios, conferencias brindadas por autoridades y profesionales expertos de los tres países, realizando además visita de observación a varias instituciones y dependencias académicas del campo de la salud y educación.

En desarrollo se encuentra actualmente la etapa que prevé el "Diseño y desarrollo del simulador" y la "Construcción de las bases curriculares regionales" que contempla la elaboración del plan de estudios, de materiales didácticos, el desarrollo de casos de simulación, la elaboración de recursos audiovisuales y la preparación de un curso de formación modular para el personal y finalizará en el 2017 con la implementación de una Maestría en Gestión Hospitalaria en la Facultad de Postgrado de la UNIBE.

El simulador de gestión sanitaria contribuirá a optimizar las competencias para el manejo de los recursos y los procesos de cada servicio de salud, en pos de obtener el mejor resultado en términos de calidad y eficiencia de las prestaciones, así como en satisfacción de los usuarios (internos y externos) y calidad de vida de los pacientes.

Permitirá a planificadores, reguladores, estudiantes y administradoresgerentes, de los diversos niveles de gestión de los Hospitales y Centros de Salud de Europa y América Latina, analizar a través de modelos matemáticos, y en entornos virtuales colaborativos, los diversos resultados que pueden obtenerse a partir de modificaciones en las múltiples variables de estructura (presupuestos, recursos humanos, equipamiento, insumos) y de procesos (asistenciales y no asistenciales) que son responsables de la reconocida complejidad de la gestión sanitaria.

El proyecto contribuirá a mejorar el acceso a la Educación superior de los profesionales que trabajan en las instituciones de la salud a través del desarrollo e implementación de un nuevo plan de estudios en la gestión de la asistencia sanitaria mediante la educación a distancia y la formación basada en la simulación y por otro lado, mejorar el nivel de competencias y habilidades para el diseño e implementación de planes de estudios de postgrado con componentes de e-learning y la formación basada en la simulación y será de suma importancia considerando que no existen registros previos a nivel global de una invención de alcance similar aplicada al sector sanitario. 